GUEST EDITORIAL

\title{
Evolving concepts of stroke and stroke management in South Africa: Quo vadis?
}

\section{Definition of stroke}

Stroke is traditionally defined as a neurological deficit attributed to an acute focal injury of the central nervous system (CNS), which occurs on a vascular basis. Stroke subtypes include ischaemic stroke/cerebral infarction, intracerebral haemorrhage (ICH) and subarachnoid haemorrhage (SAH). Stroke is a major cause of disability and death worldwide. Despite its global impact, the term stroke is not consistently defined in clinical practice, in clinical research or in assessment of the public's health. Advances in basic science, neuropathology, and neuroimaging have improved the understanding of ischaemia, infarction, and haemorrhage in the CNS. ${ }^{[1]}$ Updated definitions of stroke and stroke subtypes are provided in Box 1.

\section{Stroke classification and aetiology}

Stroke is classified into two major types: brain ischaemia and brain haemorrhage (due to ICH and SAH). Ischaemia followed by recirculation into an infarcted area may convert into haemorrhagic infarcts and may aggravate cerebral oedema owing to disruption of the blood-brain barrier. Acute ischaemic strokes (AIS) may be secondary to atherosclerosis, thrombosis (i.e. local in situ obstruction of an artery from arteriosclerosis, dissection or fibromuscular dysplasia), embolism (i.e. particles of debris originating elsewhere that block arterial access to a particular brain region) or systemic hypoperfusion.
Blood disorders causing coagulopathy are an uncommon primary cause of stroke.

Transient ischaemic attack (TIA) is traditionally defined clinically by the temporary nature of the associated neurological symptoms, which last $<24$ hours owing to neurological dysfunction caused by focal brain, spinal cord or retinal ischaemia, without acute infarction. The definition is changing with recognition that transient neurological symptoms are frequently associated with permanent brain tissue injury and the differentiation between TIA and AIS is arbitrary, as the two entities share the same spectrum.

The causes of AIS include:

- Large-vessel disease - atherosclerosis, dissection, Takayasu arteritis, giant-cell arteritis and fibromuscular dysplasia.

- Small-vessel disease - lipohyalinosis, fibrinoid degeneration and microatheroma formation.

- Cardioembolic disease - atrial fibrillation and paroxysmal atrial fibrillation, rheumatic mitral or aortic valve disease, bioprosthetic and mechanical heart valves, atrial or ventricular thrombus, sick sinus syndrome, sustained atrial flutter, recent myocardial infarction (within 1 month), chronic myocardial infarction together with ejection fraction $<28 \%$, symptomatic congestive heart failure with ejection fraction $<30 \%$, dilated cardiomyopathy, Libman-Sacks endocarditis, infective endocarditis, papillary fibro-elastoma, left atrial myxoma and coronary artery bypass graft surgery.

Box 1. Definition of stroke

The term stroke should be broadly used to include all of the following definitions:

CNS infarction. Brain, spinal cord or retinal cell death attributable to ischaemia, based on: (i) pathological, imaging or other objective evidence of cerebral, spinal cord or retinal focal ischaemic injury in a defined vascular distribution; or (ii) clinical evidence of cerebral, spinal cord or retinal focal ischaemic injury based on symptoms persisting $\geq 24$ hours or until death, excluding other aetiologies. (Note: CNS infarction includes haemorrhagic infarctions, types I and II.)

Ischaemic stroke. An episode of neurological dysfunction caused by focal cerebral, spinal or retinal infarction. (Note: evidence of CNS infarction is defined above.)

Silent CNS infarction. Imaging or neuropathological evidence of CNS infarction, without a history of acute neurological dysfunction attributable to the lesion.

Intracerebral haemorrhage. A focal collection of blood within the brain parenchyma or ventricular system that is not caused by trauma. (Note: intracerebral haemorrhage includes parenchymal haemorrhages after CNS infarction, types I and II.)

Stroke caused by intracerebral haemorrhage. Rapidly developing clinical signs of neurological dysfunction attributable to a focal collection of blood within the brain parenchyma or ventricular system that is not caused by trauma.

Silent cerebral haemorrhage. A focal collection of chronic blood products within the brain parenchyma, subarachnoid space or ventricular system on neuroimaging or neuropathological examination that is not caused by trauma and without a history of acute neurological dysfunction attributable to the lesion.

Subarachnoid haemorrhage. Bleeding into the subarachnoid space (the space between the arachnoid membrane and the pia mater of the brain or spinal cord).

Stroke caused by subarachnoid haemorrhage. Rapidly developing signs of neurological dysfunction and/or headache because of bleeding into the subarachnoid space (the space between the arachnoid membrane and the pia mater of the brain or spinal cord), which is not caused by trauma.

Stroke caused by cerebral venous thrombosis. Infarction or haemorrhage in the brain, spinal cord or retina because of thrombosis of a cerebral venous structure. Symptoms or signs caused by reversible oedema without infarction or haemorrhage do not qualify as stroke.

Stroke, not otherwise specified. An episode of acute neurological dysfunction presumed to be caused by ischaemia or haemorrhage, persisting $\geq 24$ hours or until death, but without sufficient evidence to be classified as one of the above. 
- Systemic hypoperfusion - cardiac pump failure caused by cardiac arrest, arrhythmia, acute myocardial ischaemia, pulmonary embolism, pericardial effusion or bleeding. Hypoxaemia may further reduce the amount of oxygen carried to the brain.

- Venous sinus thrombosis.

- Blood disorders - sickle cell anaemia, polycythaemia vera, essential thrombocytosis, heparin-induced thrombocytopenia, protein $\mathrm{C}$ or $\mathrm{S}$ deficiency (acquired or congenital), prothrombin gene mutation, factor V Leiden (resistance to activated protein C), antithrombin III deficiency, antiphospholipid syndrome and hyperhomocysteinaemia.

\section{Stroke epidemiology}

Globally, the proportion of strokes due to ischaemia is $68 \%$, while haemorrhagic stroke (ICH and SAH combined) accounts for $32 \%$. There is a higher incidence of haemorrhagic stroke in low- and middle-income countries (LMICs). ${ }^{[2]}$ In the USA, as in many highincome settings, the proportion of all strokes due to ischaemia approaches $90 \% \cdot{ }^{[3]}$ Worldwide, stroke is the second most common cause of mortality and the third most common cause of disability. ${ }^{[4]}$ In China, which has the greatest burden of stroke in the world, the agestandardised prevalence, incidence and mortality rates are estimated to be 1115,247 and 115 per 100000 person-years, respectively. ${ }^{[5]}$ The overall rate of stroke-related mortality is decreasing in high- and low-income countries, but the absolute number of people with stroke, stroke survivors, stroke-related deaths, and the global burden of stroke-related disability are high and increasing. ${ }^{[6]}$ Men have a higher incidence of stroke than women at younger but not older ages, with the incidence reversed and higher for women by $\geq 75$ years of age. ${ }^{[3]}$ In the USA, blacks have an increased risk of stroke compared with whites. $^{[7]}$

In 2010 , stroke was responsible for 5.3 million deaths or $10 \%$ of deaths worldwide. ${ }^{[8]}$ The absolute number of people affected by stroke has been increasing yearly since 1990, along with the numbers of disabled stroke survivors and stroke-related deaths. ${ }^{[2]}$ If current trends continue, by 2030 there will be 20 million annual deaths caused by stroke and 70 million stroke survivors worldwide. More than $80 \%$ of the stroke burden occurs in LMICs; yet, reliable data on stroke epidemiology, especially incidence and morbidity, are scarce in these settings, particularly in many African countries ${ }^{[9]}$ Epidemiological data are required to better describe trends and to develop appropriate cost-effective prevention and treatment strategies. In the absence of up-to-date estimates of the epidemiological burden of stroke, the economic impact of stroke is possibly underestimated. Global estimates, mostly derived from high-income countries, show that 3\% of total health system resources are devoted to stroke. ${ }^{[10]}$

\section{Stroke in South Africa}

Much of the population of South Africa (SA) is undergoing a rapid epidemiological transition with increased exposure to, and development of, stroke risk factors, together with ageing. In SA, stroke is responsible for $\sim 25000$ deaths annually and 95000 years lived with disability. ${ }^{[11]}$ In 2000 , stroke was the third most common cause of death in SA, after HIV/AIDS and coronary artery disease. ${ }^{[12]}$ Black women had the highest mortality rate from stroke (160 per 100000$)$, while mortality was lowest in white men (72 per 100 000). Deaths in the black population were double those in the white population. The risk of stroke increases with age: there are more stroke deaths in older South Africans, where stroke is the most common cause of death of people $>50$ years. ${ }^{[13]}$ The Southern African Stroke Prevention Initiative (SASpi) study provided the only community-based data on the prevalence of stroke in SA. The age-standardised prevalence of stroke was 290 per 100000 , and the crude prevalence in rural South Africans was 300 per $100000 .{ }^{[14]}$ Stroke prevalence in urban areas is probably higher. The SASpi study showed that there were far more disabled stroke survivors in rural SA than in Tanzania, and a similar number in New Zealand, which has a high-income population with (most likely) a higher incidence of stroke than SA. ${ }^{[14]}$ More recently, a crude stroke rate of 244 per 100000 person-years was reported in the Agincourt subdistrict, Mpumalanga Province, and 1070 disabilityadjusted life years (DALYs) were lost owing to stroke, indicating very high rates of stroke incidence and disability in rural SA. ${ }^{[8]}$

The SA National Burden of Disease Study estimated DALYs and years of life lost owing to premature death and found that, despite the high prevalence of HIV/AIDS, violence and trauma, stroke is the 8th most important cause of years of life lost owing to illness, and the 9th most important cause of disability in SA. ${ }^{[12]}$ The SA Comparative Risk Assessment Collaborating Group estimated the contribution of 8 risk factors to stroke: hypertension (52\%), tobacco use (24\%), excess body weight (18\%), high cholesterol (15\%), physical inactivity (12\%), low fruit and vegetable intake (12\%), diabetes (8\%) and alcohol consumption (8\%). ${ }^{[15]}$ Stroke management, especially in young South Africans, is often complicated by the high prevalence of HIV/AIDS. ${ }^{[16]}$

In 2010, the SA Stroke Society (SASS) published guidelines on the management of stroke and TIA with the objective of placing recommendations in the current SA context, and grading existing evidence according to the level of scientific rigour. ${ }^{[17]}$

\section{Stroke management}

Most SA patients with stroke seen in primary healthcare settings struggle to regularly access basic medicine, such as aspirin, statins, and antihypertensive and antidiabetic medications. Furthermore, because of the limited number of stroke units, which are situated almost exclusively in large urban centres, there are significant disparities in the quality of care that stroke patients receive in SA. Access to reperfusion therapies is not universally accessible, even in SA urban centres.

Despite the high disease burden, some progress has been made and stroke is no longer a hopeless condition. Dedicated stroke units were the first step in improving care, with several randomised trials showing that death and handicap could be prevented in 50 patients for every 1000 admitted. ${ }^{[2,17]}$ Intravenous thrombolysis offered the next step forward, with death and disability prevented in 143 patients for every 1000 treated with intravenous recombinant tissue plasminogen activator therapy within 3 hours of symptom onset. ${ }^{[18]}$ It was later shown that the 3 -hour time window for thrombolysis administration could be increased to 4.5 hours; and, if the trial entry and exclusion criteria were met, up to $30 \%$ of all AIS patients could benefit. The most recent evolution has been mechanical thrombectomy, performed in the catheterisation laboratory, where the occluding cerebral vessel clot is extracted. Thrombectomy is a highly effective treatment, preventing death and disability in 200 patients for every 1000 treated. Treatment should not only be directed at cure; however, those who survive with disability should be offered rehabilitation helping them to recover and achieve their maximum potential, as well as reintegrate into society.

For patients to benefit from these advances, we need to get them to the correct facilities in time for treatment. This requires co-ordination of services, including emergency assessment, transfer and delivery to stroke centres. This is most effective in countries such as Switzerland, where stroke referral is legislated. Being fully informed regarding what is possible, is the first step in improving care, which is the purpose of this issue of CME. 
In this CME, 3 articles cover different aspects of stroke management. All of these services are currently available in SA and we need to ensure that all patients can access them. In the first article, Bateman ${ }^{[20]}$ comprehensively reviews the management of AIS. In the next article, Harrichandparsad ${ }^{[21]}$ gives an update on the history, current evidence, patient selection and practical aspects of mechanical thrombectomy for AIS, and in the last article, Whitehead and Baalbergen ${ }^{[22]}$ remind us of the principles of post-stroke rehabilitation. Post-stroke rehabilitation is delivered by an interdisciplinary team experienced in the rehabilitation process, with the aim of improving patient outcomes by decreasing the chance of developing secondary complications and maximising a patient's independence despite their impairments.

It is our hope that this update on stroke management will improve the management of stroke in SA.

\section{Allan Taylor}

Division of Neurosurgery, Department of Surgery, Faculty of Health Sciences, University of Cape Town and Groote Schuur Hospital, Cape Town, South Africa

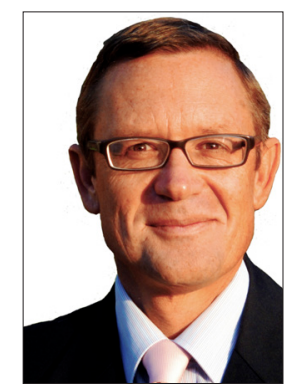

\section{Ntobeko A B Ntusi}

Division of Cardiology, Department of Medicine, Faculty of Health Sciences, University of Cape Town and Groote Schuur Hospital; Cape Universities Body Imaging Centre, Faculty of Health Sciences, University of Cape Town; and Hatter Institute for Cardiovascular Research in Africa, Department of Medicine, Faculty of Health Sciences, University of Cape Town, South Africa ntobeko.ntusi@uct.ac.za

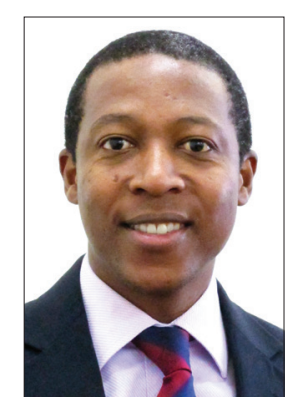

1. Sacco RL, Kasner SE, Broderick JP, et al. An updated definition of stroke for the 21st century: A statement for healthcare professionals from the American Heart Association/American Stroke Association. Stroke 2013;44(7):2064-2089. https://doi.org/10.1161/STR.0b013e318296aeca

2. Krishnamurthi RV, Feigin VL, Forouzanfar MH, et al. Global and regional burden of first-ever ischaemic and haemorrhagic stroke during 1990 - 2010: Findings from the Global Burden of Disease Study 2010. Lancet Glob Health 2013;1(5):e259-e281. https://doi.org/10.1016/S2214-109X(13)70089-5
3. Benjamin EJ, Blaha MJ, Chiuve SE, et al. Heart disease and stroke statistics - 2017 update: A report from the American Heart Association. Circulation 2017;135(10):e146-e603. https://doi.org/10.1161/ from the American Hea

4. Lozano R, Naghavi M, Foreman K, et al. Global and regional mortality from 235 causes of death for Lozano R, Naghavi M, Foreman K, et al. Global and regional mortality from 235 causes of death for
20 age groups in 1990 and 2010: A systematic analysis for the Global Burden of Disease Study 2010 . Lancet 2012;380(9859):2095-2128. https://doi.org/10.1016/S0140-6736(12)61728-0

5. Wang W, Jiang B, Sun H, et al. Prevalence, incidence, and mortality of stroke in China: Results from a nationwide population-based survey of 480687 adults. Circulation 2017;135(8):759-771. https://doi. org/10.1161/CIRCULATIONAHA.116.025250

1. Feigin VL, Forouzanfar MH, Krishnamurthi R, et al. Global and regional burden of stroke during 1990 - 2010: Findings from the Global Burden of Disease Study 2010. Lancet 2014;383(9913):245-254 https://doi.org/10.1016/S0140-6736(13)61953-4

2. White $\mathrm{H}$, Boden-Albala B, Wang $\mathrm{C}$, et al. Ischemic stroke subtype incidence among whites, blacks, and Hispanics: The Northern Manhattan Study. Circulation 2005;111(10):1327-1331. https://doi.org/10.1161/01. CIR.0000157736.19739.D0

3. Maredza M, Bertram MY, Tollman SM. Disease burden of stroke in rural South Africa: An estimate of incidence, mortality and disability adjusted life years. BMC Neurol 2015;15:54. https://doi.org/10.1186/s12883-015-0311-7

4. Sajjad A, Chowdhury R, Felix JF, et al. A systematic evaluation of stroke surveillance studies in lowand middle-income countries. Neurology 2013;80(7):677-684. https://doi.org/10.1212/WNL.0b013e$318281 \mathrm{ccc} 6 \mathrm{e}$

5. Evers SM, Struijs JN, Ament AJ, van Genugten ML, Jager JHC, van den Bos GA. International comparison of stroke cost studies. Stroke 2004;35(5):1209-1215. https://doi.org/10.1161/01.STR 0000125860.48180 .48

6. Bertram MY, Katzenellenbogen J, Vos T, Bradshaw D, Hofman KJ. The disability adjusted life year due to stroke in South Africa in 2008. Int J Stroke 2013;8(Suppl A100):76-80. https://doi.org/10.1111/ j.1747-4949.2012.00955.x

7. Alberti KG, Zimmet P, Shaw J. The International Diabetes Federation Epidemiology Task Force Consensus Group. The metabolic syndrome: A new worldwide definition. Lancet 2005;366(9491):10591062. https://doi.org/10.1016/S0140-6736(05)67402-8

8. Isozumi K. Obesity as a risk factor for cerebrovascular disease. Keio J Med 2004;53(1):7-11. https:// doi.org/10.2302/kjm.53.7

9. Pate RR, Pratt M, Blair SN, et al. Physical activity and public health: A recommendation from the Centers for Disease Control and Prevention and the American College of Sports Medicine. JAMA
(atic 1995;273(5):402-407. https://doi.org/10.1001/jama.1995.03520290054029

10. Steffen LM, Jacobs DR Jr, Stevens J, Shahar E, Carithers T, Folsom AR. Associations of whole-grain refined-grain, and fruit and vegetable consumption with risks of all-cause mortality and incident coronary artery disease and ischemic stroke: The Atherosclerosis Risk in Communities (ARIC) Study. Am J Clin Nutr 2003;78(3):383-390. https://doi.org/10.1093/ajen/78.3.383

11. Benjamin LA, Bryer A, Lucas S, et al. Arterial ischemic stroke in HIV: Defining and classifying etiology for research studies. Neurol Neuroimmunol Neuroinflamm 2016;3(4):e254. https://doi.org/10.1212 NXI.0000000000000254

12. Bryer A, Connor MD, Haug P, et al. South African guideline for management of ischaemic stroke and transient ischaemic attack 2010: A guideline from the South African Stroke Society (SASS) and the SASS Writing Committee. S Afr Med J 2010;100(11):747-778.

13. Emberson J, Lees KR, Lyden P, et al. Effect of treatment delay, age, and stroke severity on the effects of intravenous thrombolysis with alteplase for acute ischaemic stroke: A meta-analysis of individual patient data from randomised trials. Lancet 2014;384(9958):1929-1935. https://doi.org/10.1016/ patient data from rand
S0140-6736(14)60584-5

14. Goyal M, Menon BK, van Zwam WH, et al. Endovascular thrombectomy after large-vesse ischaemic stroke: A meta-analysis of individual patient data from five randomised trials. Lancet 2016;387(10029):1723-1731. https://doi.org/10.1016/S0140-6736(16)00163-X

15. Bateman K. Medical management of acute ischaemic stroke. S Afr Med J 2019;109(2):72-76. https:// doi.org/10.7196/SAMJ.2019.v109i2.00008

16. Harrichandparsad R. Mechanical thrombectomy for acute ischaemic stroke. S Afr Med J 2019;109(2):77-80. https://doi.org/10.7196/SAMJ.2019.v109i2.00010

17. Whitehead S, Baalbergen E. Post-stroke rehabilitation. S Afr Med J 2019;109(2):81-83. https://doi org/10.7196/SAMJ.2019.v109i2.00011

S Afr Med J 2019;109(2):69-71. DOI:10.7196/SAMJ.2019.v109i2.00009 